SU-ITP-01/50, SLAC-PUB-9083

PUPT-2019

hep-th/0112197

\title{
Brane/Flux Annihilation and the String Dual of a Non-Supersymmetric Field Theory
}

\author{
Shamit Kachru ${ }^{2}$, John Pearson ${ }^{1}$ and Herman Verlinde ${ }^{1}$ \\ ${ }^{1}$ Department of Physics, Princeton University, Princeton, NJ 08544 \\ ${ }^{2}$ Department of Physics and SLAC, Stanford University, Stanford, CA 94305/94309
}

\begin{abstract}
We consider the dynamics of $p$ anti-D3 branes inside the Klebanov-Strassler geometry, the deformed conifold with $M$ units of RR 3-form flux around the $S^{3}$. We find that for $p<<M$ the system relaxes to a nonsupersymmetric NS 5-brane "giant graviton" configuration, which is classically stable, but quantum mechanically can tunnel to a nearby supersymmetric vacuum with $M-p$ D3 branes. This decay mode is exponentially suppressed and proceeds via the nucleation of an NS 5-brane bubble wall. We propose a dual field theory interpretation of the decay as the transition between a nonsupersymmetric "baryonic" branch and a supersymmetric "mesonic" branch of the corresponding $S U(2 M-p) \times S U(M-p)$ low energy gauge theory. The NS 5-brane tunneling process also provides a simple visualization of the geometric transition by which D3-branes can dissolve into 3-form flux.
\end{abstract}




\section{Introduction}

The search for calculable and nontrivial nonsupersymmetric string vacua remains a problem of basic interest in string theory. In the context of AdS/CFT duality [1], this is related to the construction of gravitational "anti-holographic" descriptions for nonsupersymmetric $4 \mathrm{~d}$ quantum gauge theories. Here, we describe the construction of a simple nonsupersymmetric string background which has both a holographic description in terms of $4 \mathrm{~d}$ gauge theory and realization in the context of warped string compactifications. The model possesses several attractive features, among them the existence of certain discrete parameters which allow it to be rendered classically stable and arbitrarily long-lived and the ability to set the regime of nonsupersymmetric dynamics at energies exponentially far below the Planck scale.

One of the recent advances in the anti-holographic description of gauge theories is the discovery of several smooth gravitational duals for $\mathcal{N}=1$ supersymmetric pure Yang-Mills theory $[2,3,4]$. One such dual, as discussed by Klebanov and Strassler (KS), starts with the theory of $N$ D3 branes probing $M$ "fractional" wrapped D5 branes in a conifold geometry. In the final geometry, the branes have disappeared; they are replaced by RR and NS threeform fluxes through two intersecting three-cycles in the deformed conifold [2]. The crossed fluxes carry $N$ units of $F_{5}$-form charge, in keeping with the starting point. The holographic description of the geometry involves a $4 \mathrm{~d} S U(N+M) \times S U(N)$ gauge theory undergoing an RG cascade, in which repeated Seiberg duality transformations relate weakly-coupled descriptions of the physics. In the case $N=K M$ for some integral $K$, the IR field theory at the end of the cascade is a pure $\mathcal{N}=1$ supersymmetric $S U(M)$ Yang-Mills theory. The KS model can be embedded into a compact geometry [5] and used to realize warping as a means of generating large hierarchies as in the Randall-Sundrum scenario [6] along the lines advocated in [7] (for other discussions along these lines, see e.g. [8]). Roughly speaking, the pure Yang-Mills theory becomes the theory on the "infrared brane" in the RS description, while the string compactification manifold provides an effective "Planck brane".

In our model, along with the conifold geometry with crossed three-form fluxes, we introduce an additional ingredient which breaks the supersymmetry of the solution: We place some number $p<<M$ of anti-D3 branes at the tip of the deformed conifold. We expect that this configuration can be classically stable since, although the fluxes carry D3 brane charge, there is no immediate analogue of brane/anti-brane annihilation. Instead, the system must relax by undergoing some form of "brane/flux annihilation" in which one of the 3 -form fluxes jumps by one unit, allowing part of the D3-brane charge to materialize in the form of actual branes. Here we will identify and analyze this process in some detail; we will find that, in the regime of interest to us, it is an exponentially suppressed tunneling effect which proceeds via the nucleation of an NS 5-brane bubble wall. We will argue that, in the holographic dual description, the decay represents a transition between a metastable non-supersymmetric "baryonic" branch and a supersymmetric "mesonic" branch of the corresponding $S U(2 M-p) \times S U(M-p)$ low energy gauge theory.

The organization of this paper is as follows. In $\S 2$, we discuss preliminaries and describe 
our basic physical expectations. We also discuss the two different vacuum branches of the supersymmetric KS system, since this structure will be of importance to us later. Then, in sections $\S 3$ and $\S 4$, we study the basic dynamics of the anti-D3 branes in the conifold and flux background. We will do this from two perspectives. In $\S 3$, we will look at the flux induced potential for anti-D3 branes and present evidence that it induces them to expand into an NS 5-brane giant graviton configuration. In $\S 4$ we will then examine this system directly from the viewpoint of the NS 5-brane action with induced anti-D3 charge. By combining both perspectives and establishing a precise correspondence between them, we obtain a consistent picture where the anti-branes blow up into a fivebrane wrapping an $S^{2}$ of some fixed size on the small (A-cycle) $S^{3}$ inside the conifold. For small enough $p / M$, this describes a metastable false vacuum. In $\S 4.3$ we present the analysis of the Euclidean NS 5 brane bubble wall which mediates the quantum decay of our theory to a nearby supersymmetric vacuum with slightly different fluxes and $M-p$ D3 branes. This is the true vacuum, and is one of the models discussed in [2]. In $\S 5$ we present our best guess for the correct holographic description of our non-supersymmetric theory in terms of a long-lived false vacuum in the KS field theory. We also discuss the type IIA dual description. Finally, in section $\S 6$, we give a short discussion of the model when considered as a warped string compactification and describe some features of the $4 \mathrm{~d}$ effective supergravity theory.

As an interesting aside, we note that our study also sheds light on the geometric transition

[4] between string backgrounds with D3-branes sources and smooth KS-geometries with only 3-form flux [2]. Contrary to expectation, we will see that this transition can be understood in terms of the dynamics of the D3-branes themselves, which undergo via the formation of a "fuzzy" NS 5-brane a continuous transmutation into pure flux. This transition takes place inside supersymmetric domain walls interpolating between the two phases, the BPS domain wall solution described in $§ 4.2$.

\section{Preliminaries}

In this section, we review some of the relevant aspects of the KS geometry and its holographically dual gauge theory description. We describe our non-supersymmetric model and some of our physical expectations in $\S 2.2$.

\subsection{The Klebanov-Strassler geometry from F-theory}

In most of our investigation, we will consider the non-compact limit of the KlebanovStrassler geometry. For purposes of presentation and to facilitate later discussions of our model in the context of warped string compactifications, we will start from the point of view of F-theory with non-zero 3-form fluxes $H_{3}$ and $F_{3}$. Such models have been investigated from an M-theory and F-theory perspective in, e.g., $[9,10,5,11]$. To simplify notation, we assume as in [5] that we are working in a limit where the F-theory compactification on the 
fourfold $X$ can be viewed as an orientifold of IIB string theory on a Calabi-Yau threefold $Y$. Besides the 3-form flux, the geometry will typically involve the insertion of $N_{3}$ D3-branes and/or $\bar{N}_{3}$ anti-D3 branes. The net D3 charge

$$
Q_{3}=N_{3}-\bar{N}_{3}
$$

is fixed via the global tadpole condition

$$
\frac{\chi(X)}{24}=Q_{3}+\frac{1}{2 \kappa_{10}^{2} T_{3}} \int_{Y} H_{3} \wedge F_{3} .
$$

Here $\chi(X)$ is the Euler characteristic of the CY fourfold that specifies the F-theory compactification, and $T_{3}$ is the D3-brane tension.

We are interested in expanding about a singular point in moduli space where $Y$ has developed a conifold singularity. The KS solution corresponds to the placing of $M$ units of $F_{3}$ flux through the $A$-cycle of the conifold and $K$ units of $H_{3}$ through the dual $B$-cycle:

$$
\begin{aligned}
& \frac{1}{4 \pi^{2}} \int_{A} F_{3}=M, \\
& \frac{1}{4 \pi^{2}} \int_{B} H_{3}=-K .
\end{aligned}
$$

Assuming no other (crossed) fluxes are present, we could in principle choose $M$ and $K$ such that $\frac{\chi}{24}=M K$, so that D3 charge conservation is satisfied without the need of extra D3-brane insertions. We can therefore expect to find a smooth compactification geometry. Indeed, as discussed in detail in [5], these fluxes give rise to a non-trivial superpotential for the complex structure modulus $z_{A}=\int_{A} \Omega$ that controls the relative size of the A-cycle, stabilizing it at an exponentially small value

$$
z_{A} \sim \exp \left(-2 \pi K / M g_{\mathrm{s}}\right)
$$

As a result, the compactification geometry develops a smooth conical region, described by the warped geometry of the deformed conifold

$$
\sum_{i=1}^{4} z_{i}^{2}=\varepsilon^{2},
$$

with $\varepsilon \sim z_{A}$ exponentially small.

The exponential hierarchy (4) has a natural explanation from the holographic perspective as follows [2,5]: The geometry (5) is dual to an $S U(N+M) \times S U(N)$ gauge theory, which has a non-trivial $\beta$-function proportional to $g_{s} M$. As a result, its renormalization group flow towards the infrared involves a cascade of successive strong coupling transitions at successive scales $\mu_{n}$ with $\log \left(\mu_{n} / \mu_{n+1}\right) \sim\left(g_{s} M\right)^{-1}$. Each strong coupling transition involves a Seiberg 

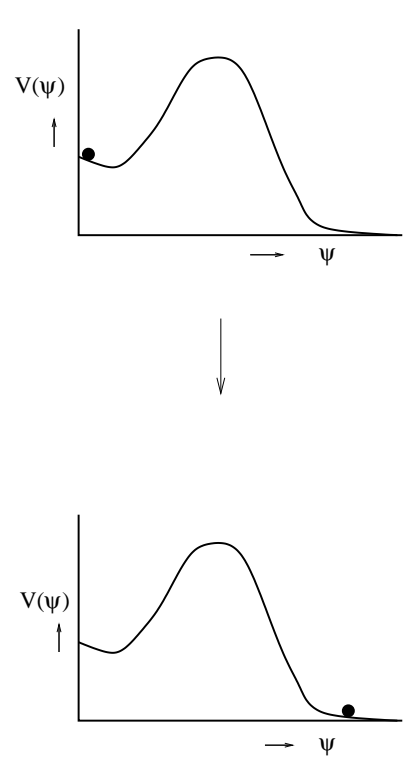
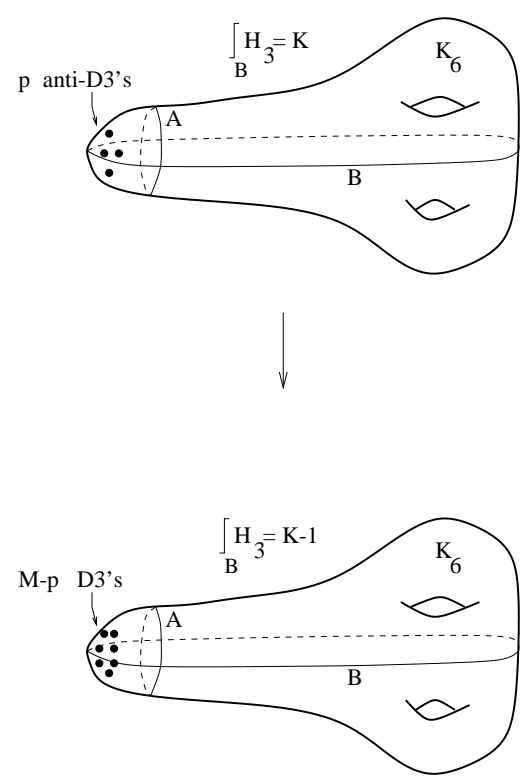

Fig 1:The decay, described in §2.2 and §4.3, takes place between an intitial nonsupersymmetric situation with $p \overline{D 3}$ branes near the tip of the conifold, to a final supersymmetric situation with $M-p$ D3-branes. The total D3-charge is preserved via the simultaneous jump in the $\mathrm{H}_{3}$ flux around the $B$-cycle by one unit, from $K$ to $K-1$.

duality transformation which lowers the rank of the larger of the two gauge groups by $2 M$. Assuming that $N=K M$, the maximal number of such dualities is $K$, leaving in the IR a pure supersymmetric $S U(M)$ Yang-Mills theory. The total range of scales traversed in this duality cascade is therefore $\log \left(\mu_{0} / \mu_{K}\right) \sim K / g_{s} M$, consistent with the supergravity prediction (4).

\subsection{Our Model and Physical Expectations}

We choose to study the model of $\S 2.1$ in the case that

$$
N_{3}=0, \bar{N}_{3}=p, \frac{\chi}{24}=K M-p
$$

with $p<<K, M$. That is, we study the theory of $p$ anti-D3 branes probing the KlebanovStrassler geometry.

This theory is nonsupersymmetric because the supersymmetry preserved by the anti-D3 branes is incompatible with the global supersymmetry preserved by the imaginary self-dual 3 -form flux of the background geometry. Thus, on general grounds, we should expect this configuration to be unstable. Indeed, if the $K M$ units of D3 charge contained in the $F_{3}$ and $H_{3}$ fluxes were realized instead as real D3 branes, we would know that the system could 
decay back to a supersymmetric KS vacuum by undergoing brane/anti-brane annihilation. In our configuration, however, there is no obvious way for this to occur.

To gain more insight, let us look at our situation (6) from the dual field theory perspective. It is natural to identify the total D3 charge carried by the compactification manifold, $\chi / 24$, with the total rank $N$ of the $S U(N+M) \times S U(N)$ dual gauge theory. Indeed, if we took $N=K M+p$, with $p<<M$, the duality cascade would proceed in $K$ steps until we were left in the infrared with an $S U(M+p) \times S U(p)$ gauge theory. As explained in [2], this theory has a moduli space parametrized by "meson" fields $N_{i j}$ which can be convincingly matched with the moduli space of $p$ D3 branes moving on the deformed conifold geometry. This is what we expect in the case $\frac{\chi}{24}=K M+p$.

Extrapolating this correspondence, we might venture that our situation (6) should be related to the KS gauge theory with $N=K M-p$. However, in this case the RG cascade can only involve $K-1$ Seiberg dualities, leaving a supersymmetric $S U(2 M-p) \times S U(M-p)$ gauge theory in the infrared. Clearly, this field theory does not correspond to the nonsupersymmetric situation (6), but instead to the supergravity solution with $M-p$ D3 branes and only $K-1$ units of $H_{N S}$ flux through the B-cycle

$$
N=M-p, \bar{N}=0, \frac{\chi}{24}=(K-1) M+(M-p)=K M-p
$$

It is a natural guess that this supersymmetric situation (7) should form the end point of a decay process beginning from the initial situation (6). Indeed, one can see that the IIB string theory admits domain walls across which the flux of $H_{3}$ through the B-cycle drops by one unit, described by NS 5 branes wrapping the A-cycle of the conifold geometry. The system (6) can therefore decay via nucleation of a bubble of supersymmetric vacuum (7) surrounded by a spherical NS 5 domain wall. This domain wall will expand exponentially as a consequence of the pressure produced by the drop in the vacuum energy. We will describe this process in much more detail in $\S 4.3$. We will find that it is a non-perturbative tunneling process for a wide range of parameters. However, this does not mean that the naive $\overline{D 3}$ description of the system is the correct description before the decay. As we will see, the $\overline{D 3}$ system also has perturbative instabilities, which cause the $\overline{D 3}$ branes to blow up into NS 5 branes wrapping spheres inside the A-cycle. Provided $p$ is sufficiently smaller than $M$, the maximum size "giant graviton" 5-brane will be a classically stable configuration. Quantum mechanically it will decay with an exponentially-suppressed probability.

To simplify our analysis, we will assume that all of the interesting dynamics takes place very close to the tip of the conifold. Indeed, it is not difficult to convince oneself that the anti-D3 branes will in general feel a net radial force $F_{r}(r)$ proportional to the 5 -form flux $F_{5}$

$$
F_{r}(r)=-2 \mu_{3} F_{5}(r)
$$

that will attract them to the tip at $r=0$. This force is a sum of gravitational and 5 -form contributions: For a $D 3$-brane these two terms cancel; in the case of $\bar{D} 3$-branes they add 
up to a net attractive force (8). Therefore, even if we began with a more generic initial distribution, we expect the anti-D3 branes to accumulate quickly near the apex at $r=0$.

The metric near the apex reads [12] (we work in string units, $\alpha^{\prime}=1$ )

$$
\begin{aligned}
d s^{2} & =a_{0}^{2} d x_{\mu} d x_{\mu}+g_{s} M b_{0}^{2}\left(\frac{1}{2} d r^{2}+d \Omega_{3}^{2}+r^{2} d \tilde{\Omega}_{2}^{2}\right) \\
a_{0}^{2} & \simeq \frac{\varepsilon^{4 / 3}}{g_{s} M} \quad b_{0}^{2} \approx 0.93266 .
\end{aligned}
$$

Since we assume that all physics takes place at $r=0$, our space-time has the topology $\mathbf{R}^{4} \times S^{3}$. The RR field $F_{3}=d C_{2}$ has a quantized flux around the $S^{3}$

$$
\int_{S^{3}} F_{3}=4 \pi^{2} M
$$

while in the supersymmetric background dictates $\star_{6} H_{3}=-g_{s} F_{3}$, so that

$$
d B_{6}=\frac{1}{g_{s}^{2}} \star_{10} H_{3}=-\frac{1}{g_{s}} d V_{4} \wedge F_{3}
$$

with $d V_{4}=a_{0}^{4} d^{4} x$. The dilaton field is constant, and the self-dual 5-form field vanishes at the tip.

\subsection{Branches of Moduli Space in the KS system}

Here we review one of the relevant features of the KS low energy field theory, following section 7 of [2]. This will be important in understanding our proposal for the holographic field theory description of the metastable false vacuum, as well as of the tunneling process that describes its quantum decay.

Consider the RG cascade of the $\mathrm{KS}$ gauge theory with $N=K M$ in its penultimate step. In this case, the unbroken gauge group is $S U(2 M) \times S U(M)$, and the $S U(2 M)$ factor has an equal number of flavors and colors. There is also a quartic superpotential whose rough form is

$$
W=\lambda\left(A_{i} B_{j} A_{k} B_{l}\right) \epsilon^{i k} \epsilon^{j l}
$$

where the $A_{i}$ are in the $(2 M, \bar{M})$ representation, the $B_{i}$ are in the conjugate representation, and $i=1,2$. We know from the analysis of [13] that in $\mathcal{N}=1$ supersymmetric QCD with $N_{f}=N_{c}$, the moduli space is quantum mechanically modified. Treating the $S U(M)$ as a global symmetry (i.e. taking its dynamical scale to vanish), this situation reduces to the one studied in [13] (with the added complication of the quartic tree-level superpotential). 
Define the "meson" fields $N_{i j, \alpha \beta}$ and the "baryon" fields $\mathcal{B}, \tilde{\mathcal{B}}$

$$
N_{i j, \alpha \beta}=A_{i, \alpha} B_{j, \beta}, \quad \mathcal{B}=\left(A_{1}\right)^{M}\left(A_{2}\right)^{M}, \quad \tilde{\mathcal{B}}=\left(B_{1}\right)^{M}\left(B_{2}\right)^{M}
$$

where $\alpha$ and $\beta$ are $S U(M)$ "flavor" indices. In order to reproduce the quantum modified moduli space of the $S U(2 M)$ theory, we should add a Lagrange multiplier term to (12)

$$
W=\lambda\left(N_{i j, \alpha \beta} N_{k l}^{\alpha \beta}\right) \epsilon^{i k} \epsilon^{j l}+X\left(\operatorname{det}(N)-\mathcal{B} \tilde{\mathcal{B}}-\Lambda^{4 M}\right)
$$

where the determinant is understood to be that of a $2 M \times 2 M$ matrix (coming from the $i, j$ and color indices on $N$ ).

There are distinct "mesonic" and "baryonic" branches of supersymmetric vacua arising from the superpotential (14). On the baryonic branch

$$
X=N=0, \quad \mathcal{B}=\tilde{\mathcal{B}}=i \Lambda^{2 M}
$$

On this branch, the $S U(M)$ factor in the gauge group remains unbroken, and one is left with pure $\mathcal{N}=1$ gauge theory in the IR. However, there is also a branch where $\mathcal{B}=\tilde{\mathcal{B}}=0$ and the mesons have non-vanishing VEVs.

$$
\operatorname{det}(N)=\Lambda^{4 M}, \quad \mathcal{B}=\tilde{\mathcal{B}}=0
$$

Now the gauge group is generically Higgsed, and there is a moduli space of vacua consisting of the theory of $M$ D3 branes probing a deformed conifold geometry.

The fact that the mesonic and baryonic branches of moduli space are disconnected is a result of the tree level superpotential (12). In the theory without (12), the quantum moduli space is defined by the equation $\operatorname{det}(N)-\mathcal{B} \tilde{\mathcal{B}}=\Lambda^{4 M}$ and one can smoothly interpolate between these branches, via a continuous path of supersymmetric vacua. In the present case, on the other hand, field configurations that interpolate between the two branches take the form of localized supersymmetric domain walls with non-zero energy density. These domain walls solve BPS equations of the form

$$
\partial_{z} \Phi^{I}=g^{I J} \partial_{J} W(\Phi)
$$

with $\Phi^{I}=\left\{N_{i j}^{\alpha \beta}, \mathcal{B}, X\right\}$, and $z$ the coordinate transverse to the domain wall. The wall tension is proportional to

$$
|\Delta W|=\left|W\left(\Phi_{b}\right)-W\left(\Phi_{m}\right)\right|,
$$


where $\Phi_{b}$ and $\Phi_{m}$ denote the respective vacuum values (15) and (16) of the two branches. By considering e.g. the special mesonic vacuum where all meson fields have the same expectation value (i.e. independent of their "flavor" index), we deduce

$$
|\Delta W|=2 \lambda M \Lambda^{4} .
$$

In $\S 4.2$ we will consider the dual supergravity description of the domain wall. In this case it will represent the transition from the smooth deformed conifold with only flux (corresponding to the baryonic branch) to the situation with one less unit of NS 3-form flux and $M$ D3-branes. In $\S 5$ we will use this dual understanding of the supersymmetric domain wall to motivate a similar holographic description of our non-supersymmetric $\overline{D 3}$ background and its decay process. Here we will similarly argue that there are two relevant branches: The first, the analogue of the baryonic branch, will be the (now nonsupersymmetric) metastable vacuum; and the second, the analogue of the mesonic branch, will be the theory with $M-p$ D3 branes probing the deformed conifold (7).

\section{The $\overline{D 3}$ brane Perspective}

We are interested in the dynamics of $p \overline{D 3}$ branes sitting at the end of the KS throat, under the influence of the $F_{3}$ and $H_{3}$ fluxes (3). We will do our analysis within the probe approximation, taking the KS background as fixed, while ignoring the backreaction due to the $\overline{D 3}$ branes. The characteristic size of the geometry is set by $R \simeq \sqrt{g_{s} M}$, while we can estimate that the backreaction due to the $p$ anti-branes extends over a region of order $r \simeq \sqrt{g_{s} p}$. Hence the distortion of deformed conifold due to presence of the $\overline{D 3}$ branes remains small as long as $p \ll M$. We will assume that we are in this regime.

It is now rather well understood how, in a non-trivial flux background, $p$ D3-branes can expand to form a spherical D5-brane (this phenomenon played an important role in the analysis of [14], for example). Here, due to specific form of the background fluxes, we will need to consider the S-dual phenomenon, where the $\overline{D 3}$ branes expand into wrapped NS 5 branes. A technical difficulty, however, is that it is not yet known how to consistently couple the background $B_{6}$-flux (11) to the matrix Born-Infeld action of the $\overline{D 3}$ branes. (This problem is directly related to that of finding a Matrix theory description of the transverse NS 5-brane.) A way around this obstacle, is to use the S-dual description of the $\overline{D 3}$ worldvolume. In $\S 4$, we will turn things around, and view things from the perspective of a wrapped NS 5 brane with $\overline{D 3}$ charge $p$ coming from a world-volume magnetic flux.

Before getting started, we need to warn the reader that strictly speaking, since $g_{s}$ is assumed to be small and thus the S-dual coupling $\tilde{g}_{s}=1 / g_{s}$ large, we are far outside of the regime of validity of the S-dual Born-Infeld action. We will nonetheless proceed with using it; in $\S 4$ will find a posteriori justification of our description when we establish a precise match with the results obtained from the NS 5-brane perspective. 


\subsection{Dielectric $\overline{D 3}$ Branes}

The worldvolume action of the $p$ anti-D3 branes, placed at the apex of the deformed conifold and in the S-dual frame, is given by the Born-Infeld action

$$
S_{B I}=\frac{\mu_{3}}{g_{s}} \int \operatorname{Tr} \sqrt{\operatorname{det}\left(G_{\|}+2 \pi g_{s} F\right) \operatorname{det}(Q)}-2 \pi \mu_{3} \int \operatorname{Tr} \mathbf{i}_{\Phi} \mathbf{i}_{\Phi} B_{6}
$$

Here

$$
Q_{j}^{i}=\delta_{j}^{i}+\frac{2 \pi i}{g_{s}}\left[\Phi^{i}, \Phi^{k}\right]\left(G_{k j}+g_{s} C_{k j}\right)
$$

Because we are working in the S-dual frame, relative to the usual Born-Infeld action, we replaced $B_{2}$ by $C_{2}$ and $C_{6}$ by $B_{6}$. The two-form $F$ here denotes the non-abelian field strength on the $\overline{D 3}$ brane worldvolume.

The scalar fields $\Phi$ parametrize the transverse location $X$ of the $\overline{D 3}$ branes, via the relation $\Phi=2 \pi X$. By making these matrix coordinates non-commutative, the anti D3branes can collectively represent a 5-dimensional brane which can be identified with the NS 5-brane. The topology of this "fuzzy NS 5 brane" is $\mathbf{R}^{4} \times S^{2}$, where the two-sphere $S^{2}$ has an approximate radius $R$ equal to

$$
R^{2} \simeq \frac{4 \pi^{2}}{p} \operatorname{Tr}\left(\left(\Phi^{i}\right)^{2}\right)
$$

It is instructive to look for the non-commutative solution for $p \ll M$, in which case $\Phi$ remains small relative to the radius of curvature of the surrounding space-time and variations in the 3 -form field strengths. In this case we may write $C_{k j} \sim \frac{2 \pi}{3} F_{k j l} \Phi^{l}$, and locally we may approximate the metric in the compact space by the flat metric $G_{k j}=\delta_{k j}$. We find that

$$
Q_{j}^{i}=\delta_{j}^{i}+\frac{2 \pi i}{g_{s}}\left[\Phi^{i}, \Phi_{j}\right]+i \frac{4 \pi^{2}}{3} F_{k j l}\left[\Phi^{i}, \Phi^{k}\right] \Phi^{l} .
$$

So we can expand

$$
\operatorname{Tr} \sqrt{\operatorname{det}(Q)} \simeq p-i \frac{2 \pi^{2}}{3} F_{k j l} \operatorname{Tr}\left(\left[\Phi^{k}, \Phi^{j}\right] \Phi^{l}\right)-\frac{\pi^{2}}{g_{s}^{2}} \operatorname{Tr}\left(\left[\Phi^{i}, \Phi^{j}\right]^{2}\right)
$$

Furthermore, we are in an imaginary self-dual flux background where $d B_{6}=-\frac{1}{g_{s}} d V_{4} \wedge F_{3}$. In an imaginary anti self-dual flux background, the cubic terms in the full potential for the $\overline{D 3}$ worldvolume fields $\Phi$ would have to cancel (by a "no-force" condition between anti-branes 
and IASD fluxes). This logic, or alternatively, direct calculation of the second term in (20), tells us that the full potential coming from (20) in this ISD flux background will be

$$
g_{s} V_{\mathrm{eff}}(\Phi) \simeq \sqrt{\operatorname{det}\left(G_{\|}\right)}\left(p-i \frac{4 \pi^{2}}{3} F_{k j l} \operatorname{Tr}\left(\left[\Phi^{k}, \Phi^{j}\right] \Phi^{l}\right)-\frac{\pi^{2}}{g_{s}^{2}} \operatorname{Tr}\left(\left[\Phi^{i}, \Phi^{j}\right]^{2}\right)+\ldots\right)
$$

As in [15], this potential has extrema away from the origin $\Phi=0$. To get some intuition, let us set $F_{k j l}=f \epsilon_{k j l}$, where we make the approximation that the A-cycle $S^{3}$ is large (which is good in the limit of large $\left.g_{s} M\right)$. The magnitude of $f$ can be read off from the normalization of the integrated RR flux as in (3), which requires

$$
f \simeq \frac{2}{b_{0}^{3} \sqrt{g_{s}^{3} M}}
$$

Demanding that $\frac{\partial V(\Phi)}{\partial \Phi^{i}}=0$ yields the equation of motion

$$
\left[\left[\Phi^{i}, \Phi^{j}\right], \Phi^{j}\right]-i g_{s}^{2} f \epsilon_{i j k}\left[\Phi^{j}, \Phi^{k}\right]=0
$$

To solve (27), notice that if one takes constant matrices $\Phi^{i}$ satisfying the commutation relations

$$
\left[\Phi^{i}, \Phi^{j}\right]=-i g_{s}^{2} f \epsilon_{i j k} \Phi^{k}
$$

then (27) is automatically satisfied. But, up to rescaling the $\Phi,(28)$ are just the commutation relations which are satisfied by a $p \times p$ dimensional matrix representation of the $S U(2)$ generators

$$
\left[J^{i}, J^{j}\right]=2 i \epsilon_{i j k} J^{k}
$$

So by setting $\Phi^{i}=-\frac{1}{2} g_{s}^{2} f J^{i}$, with $J^{i}$ the generators of any p-dimensional $S U(2)$ representation, we find solutions of (28).

Which solution is energetically preferred? Using the known value of the quadratic Casimir $c_{2}=\operatorname{Tr}\left(\left(J^{i}\right)^{2}\right)$ in each $p$-dimensional $S U(2)$ representation, one can see that the energetically preferred solution is to take the $p$-dimensional irreducible representation of $S U(2)$, for which one finds

$$
\begin{aligned}
V_{\mathrm{eff}} & \simeq \frac{\mu_{3}}{g_{s}}\left(p-\frac{\pi^{2}}{6} g_{s}^{8} f^{4} p\left(p^{2}-1\right)\right) \\
& \simeq \frac{\mu_{3} p}{g_{s}}\left(1-\frac{8 \pi^{2}\left(p^{2}-1\right)}{3 b_{0}^{12} M^{2}}\right) .
\end{aligned}
$$


The negative term in (30) comes about through a competition between the (positive) quartic term and the (negative) cubic term in (25). The other $p$-dimensional reducible representations occur as metastable vacua of (25), where the $\overline{D 3}$ branes have blown up to a number of "less giant" NS 5 branes but can still satisfy $V_{\text {eff }}<p / g_{s}$. These separate 5 -branes all want to cluster together to form the "most giant" NS 5-brane, with minimal energy equal to (30).

It is interesting to compare the energy in the giant graviton vacuum (30) to the final energy in the supersymmetric ground state, $V=0$. We see that $V_{\text {eff }}>0$ implies that $p \ll M$, which is the condition we have chosen to impose. In this regime, we find a selfconsistent picture: the $\overline{D 3}$ branes are driven by a perturbative instability to expand into an NS 5 brane wrapped on an $S^{2}$ in the A-cycle, and must await a non-perturbative effect to decay to the supersymmetric vacuum. As we will discuss in $\S 4$, the complementary analysis in terms of the NS 5 brane worldvolume action indicates that, for sufficiently large $\frac{p}{M}$, the decay to a final supersymmetric state can occur without the intermediate metastable false vacuum. Based on the above story, we can obtain a reasonable estimate for the onset of this classical instability by considering the radius $R$ (given in eqn (22)) of the fuzzy NS 5-brane in comparison to the radius $R_{0}=b_{0} \sqrt{g_{s} M}$ of the $S^{3}$

$$
R^{2} \simeq \frac{4 \pi^{2}\left(p^{2}-1\right)}{b_{0}^{8} M^{2}} R_{0}^{2}
$$

We see that there is a classical minimum only if $p / M$ is sufficiently smaller than $b_{0}^{4} / 2 \pi$; otherwise the radius of the NS 5-brane will get too close to $R_{0}$ and the configuration will become classically unstable.

\section{The NS 5-brane perspective}

In this section, we take the perspective of an NS 5-brane moving near the tip of the conifold geometry. The 5-brane is wrapped on a two-sphere $S^{2}$ inside the internal $S^{3}$ and carries $p$ units of world-volume two-form flux which induce $\overline{D 3}$ charge. As noted previously, it is a point of some concern that the NS 5 world-volume description used below has only limited validity for small sizes of the $S^{2}$. For sufficiently large $S^{2}$ radius, however, the NS 5 world-volume curvature is small compared to the string scale and one may expect that the description as given below becomes reasonably accurate.

\subsection{Giant Graviton 5-brane}

Consider an NS 5-brane of type IIB string theory located at an $S^{2}$ inside $S^{3}$ with radius specified by a polar angle $\psi$. The bosonic worldvolume action reads [14]

$$
S=\frac{\mu_{5}}{g_{s}^{2}} \int d^{6} \xi\left[-\operatorname{det}\left(G_{\|}\right) \operatorname{det}\left(G_{\perp}+2 \pi g_{s} \mathcal{F}\right)\right]^{1 / 2}+\mu_{5} \int B_{6}
$$




$$
2 \pi \mathcal{F}_{2}=2 \pi F_{2}-C_{2}
$$

This action has been obtained by S-duality from that of the D5-brane. Here $F_{2}=d A$ is the two-form field strength of the world-volume gauge field, $G_{\perp}$ denotes the induced metric along the internal $S^{2}$, and $G_{\|}$encodes the remaining components along the $d \psi$ and $\mathbf{R}^{4}$ directions. Using the explicit form (9) of the metric, we have

$$
\begin{aligned}
& d s_{\text {induced }}^{2}=b_{0}^{2} g_{s} M\left[d x_{\mu} d x^{\mu}+d \psi^{2}+\sin ^{2} \psi d \Omega_{2}^{2}\right] \\
& =\quad d s_{\|}^{2}+d s_{\perp}^{2}
\end{aligned}
$$

where (relative to eqn (9)) we have absorbed the factor of $a_{0} / R_{0}$ into $x^{\mu}$. (This means that from now on, all time and distance scales in the $\mathbf{R}^{4}$ directions are measured in red-shifted string units, or in holographic dual terminology, in terms of the dimensional transmutation scale $\Lambda$ of the low energy gauge theory.) We can evaluate the following integrals over $S^{2}$

$$
\begin{aligned}
\int_{S^{2}} \sqrt{\operatorname{det} G_{\perp}} & =4 \pi b_{0}^{2} g_{s} M \sin ^{2} \psi, \\
\int_{S^{2}} C_{2}(\psi) & =4 \pi M\left(\psi-\frac{1}{2} \sin (2 \psi)\right), \\
2 \pi \int_{S^{2}} F_{2} & =4 \pi^{2} p .
\end{aligned}
$$

This last equation reflects the fact that the NS 5-brane carries $\overline{D 3}$ brane charge $p$. Combining (36)-(38) gives:

$$
\begin{gathered}
\int_{S^{2}} \sqrt{\operatorname{det}\left(G_{\perp}+2 \pi g_{s} \mathcal{F}\right)}=4 \pi^{2} M g_{s} V_{2}(\psi) \\
V_{2}(\psi)=\frac{1}{\pi} \sqrt{b_{0}^{4} \sin ^{4} \psi+\left(\pi \frac{p}{M}-\psi+\frac{1}{2} \sin (2 \psi)\right)^{2}} .
\end{gathered}
$$

Adding the $\mu_{5} \int B_{6}$ term, obtained from eqn (11), gives a total NS 5-brane action

$$
\begin{gathered}
S=\int d^{4} x \sqrt{-\operatorname{det} G_{\|}} \mathcal{L}(\psi), \\
\mathcal{L}(\psi)=A_{0}\left(V_{2}(\psi) \sqrt{1-\dot{\psi}^{2}}-\frac{1}{2 \pi}(2 \psi-\sin 2 \psi)\right),
\end{gathered}
$$

with

$$
A_{0}=\frac{4 \pi^{2} \mu_{5} M}{g_{s}}=\frac{\mu_{3} M}{g_{s}} .
$$



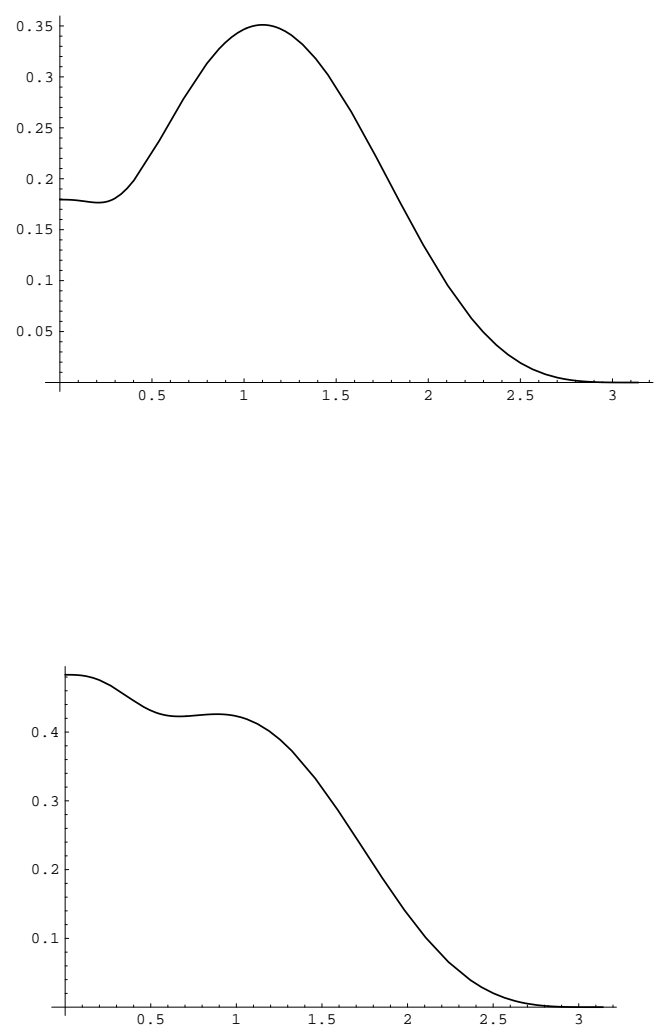

Fig 2: The effective potential $V_{\text {eff }}(\psi)$ for $\frac{p}{M} \simeq 3 \%$, showing the stable false vacuum, and for $\frac{p}{M} \simeq 8 \%$, with only a marginally stable minimum.

We can use this action to introduce a canonical momentum $P_{\psi}$ conjugate to $\psi$, and write the resulting Hamiltonian density $\mathcal{H}$ as

$$
\mathcal{H}\left(\psi, P_{\psi}\right)=-\frac{A_{0}}{2 \pi}(2 \psi-\sin 2 \psi)+\sqrt{A_{0}^{2}\left(V_{2}(\psi)\right)^{2}+P_{\psi}^{2}}
$$

which generates the time evolution of $\psi(t)$. For the moment, however, we are just interested in finding whether there exists a static solution corresponding to the "giant graviton" of $\S 3$.

Some useful intuition is obtained by considering the effective potential

$$
\begin{aligned}
V_{\text {eff }}(\psi) & \equiv \mathcal{H}\left(\psi, P_{\psi}=0\right) \\
& =A_{0}\left(V_{2}(\psi)-\frac{1}{2 \pi}(2 \psi-\sin 2 \psi)\right)
\end{aligned}
$$


In figure 2 we have plotted this effective potential $V_{\text {eff }}(\psi)$ for two different values of $p$ relative to $M$ : the top graph corresponds to $p / M \simeq 3 \%$, and shows a stable false vacuum, and the lower graph corresponds to the special case $p / M \simeq 8 \%$ at which there is only a marginally stable intermediate minimum. For $p / M>8 \%$ the slope of the effective potential is everywhere negative.

In both cases we can draw an interesting conclusion. In the regime $p / M>8 \%$, the nonsupersymmetric configuration of $p \overline{D 3}$ branes relaxes to the supersymmetric minimum via a classical process: The anti-branes cluster together to form the maximal size "fuzzy" NS 5-brane which rolls down the potential until it reduces to $M-p$ D3-branes located at the north-pole at $\psi=\pi$. In the regime with $p / M$ (sufficiently) smaller than $8 \%$, the branes reach a meta-stable state, the fuzzy NS-5 brane located at the location $\psi_{\min }$ for which $\frac{\partial V_{\text {eff }}}{\partial \psi}\left(\psi_{\min }\right)=0$. This configuration is classically stable, but will decay via quantum tunneling. We will study this tunneling process in $§ 4.3$. In both cases, the end result of the process is $M-p$ D3-branes in place of $p$ anti-D3-branes with the $H_{3}$ flux around the B-cycle changed from $K$ to $K-1$.

Now let us check the correspondence with the non-abelian description of $\S 3$. For small values of $\psi$ we can expand

$$
V_{\mathrm{eff}}(\psi) \simeq A_{0}\left(\frac{p}{M}-\frac{4}{3 \pi} \psi^{3}+\frac{b_{0}^{4} M}{2 \pi^{2} p} \psi^{4}\right)
$$

which has a minimum at $\psi_{\min }=\frac{2 \pi p}{b_{0}^{4} M}$ equal to

$$
V_{\mathrm{eff}}\left(\psi_{\min }\right) \simeq \frac{\mu_{3} p}{g_{s}}\left(1-\frac{8 \pi^{2} p^{2}}{3 b_{0}^{12} M^{2}}\right)
$$

in exact agreement with the value (30) found earlier. Moreover, the size $R \simeq \psi_{\text {min }} R_{0}$ (with $R_{0}$ the $S^{3}$ radius) of the NS 5 brane "giant graviton" exactly matches with our earlier result (32).

Another quantitative confirmation of the result (45) for the effective potential is that the difference in vacuum energy between the south and north-pole is equal to twice the tension of the anti-D3 branes

$$
V_{\text {eff }}(0)-V_{\text {eff }}(\pi)=\frac{2 p \mu_{3}}{g_{s}}
$$

This is the expected result. One way to understand this [16] is to compare our nonsupersymmetric model with a hypothetical situation with all $p$ anti-D3 branes replaced by $-p$ D3-branes, i.e. branes with opposite charge and tension as D3-branes. This last situation would preserve supersymmetry and would therefore have zero vacuum energy. To change it back to our physical situation, however, one needs to add back $p$ D3/anti-D3 pairs, with 
zero charge but with total tension $2 p \mu_{3} / g_{s}$. Notice, however, that in order to obtain the true total vacuum energy, we need to add to the result (45) a term

$$
V_{\mathrm{tot}}(\psi)=V_{\mathrm{eff}}(\psi)+\frac{p \mu_{3}}{g_{s}}
$$

so that the supersymmetric vacuum indeed has $V_{\text {tot }}=0$. This extra contribution comes from a term $\frac{\mu_{3}}{g_{s}} \chi / 24-\int H_{3} \wedge G_{3}$ in the string action which, via the global tadpole condition, adds up to $p \mu_{3} / g_{s}$.

Finally, let us return to the validity of our description. As mentioned, this is a slightly problematic question, since both the S-dual D3-brane variables and the NS 5 world-volume theory are strongly coupled. It seems reasonable, however, that at least our main qualitative conclusions, $(i)$ the anti-D3 branes expand to a "fuzzy" NS 5 brane, $(i i)$ for small enough $p / M$, the NS 5 brane will stabilize at some $S^{2}$-radius $\psi_{\min }$ proportional to $p / M$, and (iii) for large enough $p / M$ the anti-D3/NS 5 configuration will be classically unstable, will remain unchanged in a more complete treatment. One forseeable quantitative difference, for example, is that inclusion of the backreaction of the NS 5-brane on the $S^{3}$ geometry might trigger the classical instability for smaller values of $p / M$ than found above.

\subsection{BPS Domain Wall}

As discussed in $§ 2.3$, one can consider supersymmetric domain walls that interpolate between the supersymmetric "mesonic" and "baryonic" vacuum branches in the pure KS gauge theory with $p=0$. As we now show, it is possible to write a special BPS solution to the NS 5 brane equations of motion that describes a supersymmetric domain wall between the two phases. Specifically, we assume that the mesonic vacuum is such that all meson fields $\left(N_{i j}\right)^{\alpha \beta}$ have the same expectation value. In the supergravity, this is described by the configuration of $M$ D3-branes located at the same point on the $S^{3}$, which we take to be the north-pole $\psi=\pi$.

Before we describe this domain wall solution, we note that, for general $p$, the total $\mathcal{F}_{2}$ flux through the $S^{2}$ satisfies

$$
\begin{aligned}
2 \pi \int_{S_{2}} \mathcal{F}_{2} & =4 \pi\left(\pi p-M\left(\psi-\frac{1}{2} \sin (2 \psi)\right)\right) \\
& =-4 \pi\left(\pi(M-p)-M\left(\tilde{\psi}-\frac{1}{2} \sin (2 \tilde{\psi})\right)\right)
\end{aligned}
$$

with $\tilde{\psi}=\pi-\psi$. In other words, we can think of the $\mathcal{F}$ background from the "southpole perspective" as carrying $p$ units of $\overline{D 3}$ charge, or from the "north-pole perspective" as carrying $M-p$ units of D3-charge. Notice that this implies that there must be $M-p$ units of $F_{2}$ flux placed at the north-pole, that is, $M-p$ D3-branes. Hence, in the special case that 
$p=0$, the NS 5-brane at the north-pole represents $M$ D3 branes, while at the south-pole it can simply shrink from view without a trace.

The domain wall solution corresponds to an NS 5-brane configuration described by a spatial trajectory $\psi(z)$ ( $z$ the coordinate transverse to the wall) interpolating between $\psi=\pi$ and $\psi=0$. Following the same steps as above, we find that the $z$-evolution of $\psi(z)$ is governed by the Hamilton equations $\partial_{z} \psi=\frac{\partial \mathcal{H}_{z}}{\partial P_{\psi}}$ and $\partial_{z} P_{\psi}=-\frac{\partial \mathcal{H}_{z}}{\partial \psi}$ with

$$
\mathcal{H}_{z}\left(\psi, P_{\psi}\right)=-\frac{A_{0}}{2 \pi}(2 \psi-\sin 2 \psi)+\sqrt{A_{0}^{2}\left(V_{2}(\psi)\right)^{2}-P_{\psi}^{2}}
$$

We look for a trajectory that, for large negative $z$, starts at rest at the north-pole, i.e., $\psi=\pi$ and $P_{\psi}=0$. Therefore, this solution has $\mathcal{H}=0$. Solving for $P_{\psi}$ we find

$$
P_{\psi}=b_{0}^{2} \sin ^{2} \psi
$$

We thus obtain the following first order equation for $\psi(z)$

$$
\partial_{z} \psi=-\frac{b_{0}^{2} \sin ^{2} \psi}{\psi-\frac{1}{2} \sin 2 \psi}
$$

This can be integrated to (choosing the location of the domain wall around $z \simeq 0$ )

$$
z=\frac{\psi \cot \psi}{b_{0}^{2}}
$$

The right-hand side covers the half-space from $z=-\infty$ to $z=1 / b_{0}$, where the NS 5-brane trajectory has reached the south-pole at $\psi=0$. At this point the brane has disappeared, leaving behind the pure flux KS solution. In the dual gauge theory, this is the baryonic vacuum.

It is useful to think of the domain wall as an NS 5 brane wrapped around the $S^{3} \mathrm{~A}$-cycle of the conifold, deformed near the north-pole due to the presence of the $M$ D3-branes ending on it. From this perspective, it clearly has the property of inducing a jump by one unit in the $H_{3}$ flux around the B-cycle. Indeed, if we consider two such B-cycles $B\left(z_{+}\right)$and $B\left(z_{-}\right)$ located at opposite sides of the domain wall, their difference $B\left(z_{+}\right)-B\left(z_{-}\right)$represents an (otherwise contractible) 3-cycle that surrounds the NS 5-brane once. Since the NS 5-brane acts like a magnetic source for $H_{3}$ we have

$$
\int_{B\left(z_{+}\right)} H_{3}-\int_{B\left(z_{-}\right)} H_{3}=4 \pi^{2}
$$


The tension of the domain wall is obtained by evaluating the classical action of the above solution per unit time and area. We have

$$
S=\int d t d^{2} x \sqrt{-\operatorname{det} G_{3}} T_{w a l l}
$$

with

$$
T_{\text {wall }}=A_{0} b_{0} \sqrt{g_{s} M} \int_{0}^{\pi} d \psi \frac{b_{0}^{2} \sin ^{2} \psi}{\pi}=\frac{\mu_{5} 2 \pi^{2} b_{0}^{3}\left(g_{s} M\right)^{3 / 2}}{g_{s}^{2}}
$$

As expected, this result for the domain wall tension breaks up as the product of the NS 5 -brane tension $\mu_{5} / g_{s}^{2}$ times the volume of the $S^{3}$ (with radius $R_{0}=b_{0} \sqrt{g_{s} M}$ ) wrapped by it. From a holographic viewpoint, this should be compared with the formula (19) obtained from the gauge theory effective superpotential.

We should mention that the probe approximation used here is no longer strictly valid near the north-pole $\psi=\pi$, since the $M$ D3-branes represent an appreciable stress-energy that will have a non-negligible effect on the background geometry. Previous experience with supersymmetric configurations of this kind [14], however, suggests that such backreaction effects do not significantly alter the results for quantities like the domain wall tension.

\subsection{Vacuum Tunneling}

We now turn to a description of the decay of the non-supersymmetric configuration with $p$ non-zero. This takes place via nucleation of a bubble of supersymmetric vacuum (7) surrounded by a spherical NS 5 domain wall which expands exponentially as a consequence of the pressure produced by the drop in the vacuum energy. To obtain the nucleation rate, it is standard practice to look for a corresponding Euclidean solution. The relevant solution for us is an NS-5 brane trajectory $\psi(R)$, where $R$ is the radial coordinate in $\mathbf{R}^{4}$, connecting the "giant graviton" configuration at $\psi=\psi_{\text {min }}$ at large $R$ to an instantonic domain wall located near some appropriate radius $R=R_{*}$ at which the solution reaches the supersymmetric minimum $\psi=\pi$.

The total action functional for such a trajectory reads

$$
S=B_{0} \int_{R_{*}}^{\infty} d R R^{3}\left(V_{2}(\psi) \sqrt{1+\left(\partial_{R} \psi\right)^{2}}+\frac{1}{\pi}\left(\pi \frac{p}{M}-\psi+\frac{1}{2} \sin 2 \psi\right)\right)
$$

with

$$
B_{0}=2 \pi^{2} b_{0}^{4} \mu_{3} g_{s} M^{3}
$$

As before, it is convenient to write the classical equations of motion for this action in the 

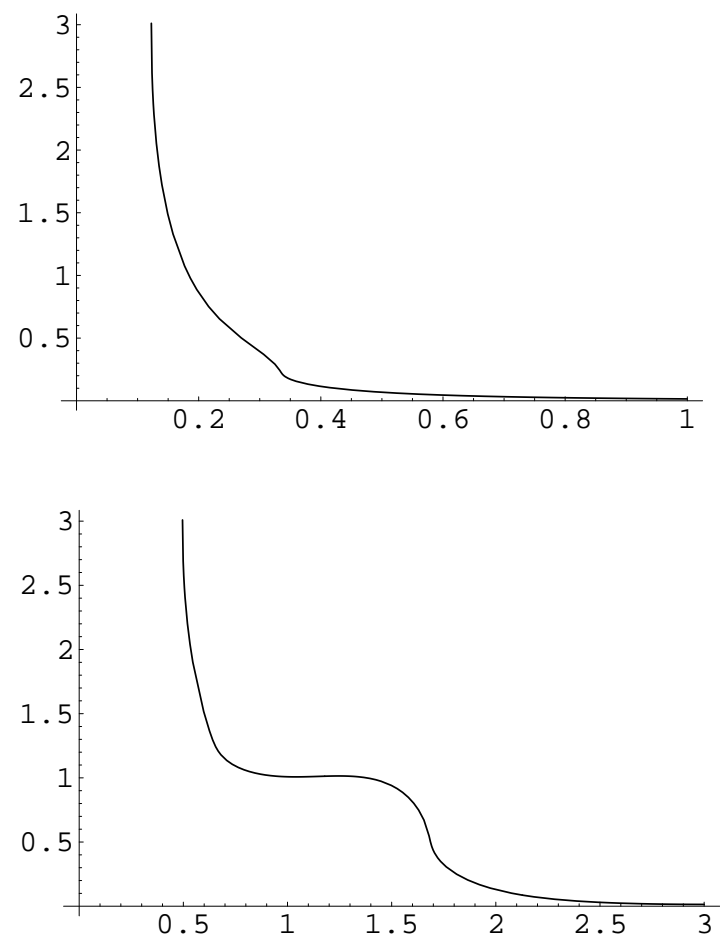

Fig 3: The Euclidean NS 5-brane trajectory $\psi(R)$ for two values of $p / M$ : the upper trajectory corresponds to $\frac{p}{M} \simeq 3 \%$, and the lower one to the near critical value $\frac{p}{M} \simeq 8 \%$.

form of Hamilton equations $\partial_{R} \psi=\frac{\partial \mathcal{H}_{R}}{\partial P_{\psi}}$ and $\partial_{R} P_{\psi}=-\frac{\partial \mathcal{H}_{R}}{\partial \psi}$ with

$$
\mathcal{H}_{R}\left(\psi, P_{\psi}\right)=-\frac{B_{0} R^{3}}{2 \pi}(2 \psi-\sin 2 \psi)+\sqrt{B_{0}^{2} R^{6}\left(V_{2}(\psi)\right)^{2}-P_{\psi}^{2}}
$$

In figure 3 we have drawn the resulting Euclidean NS 5-brane trajectories $\psi(R)$ for two values of $p / M$.

In principle, we could extract the nucleation rate from the above formulas for general $p / M$ by evaluating the total action of the (numerically obtained) classical solutions, though this is a laborous prospect. Instead, we can quite easily obtain the leading order decay rate in the limit of small $p / M$ as follows: From figure 3, the classical trajectory for small $p$ naturally divides up into two separate regions: it stays flat near the non-supersymmetric 
minimum until coming very close to the domain wall radius $R_{*}$ where it quickly moves toward the north-pole value $\psi=\pi$. On these grounds, we divide the total action into a contribution coming from the non-zero vacuum energy (given in (49) and (47)) of the nonsupersymmetric "giant graviton" at $R>R_{*}$ and a second contribution coming from the tension of the domain wall. Using the "thin wall approximation" [17] we may write

$$
S=T_{\text {wall }} \operatorname{Vol}_{3}\left(R_{*}\right)-V_{\text {tot }}\left(\psi_{\text {min }}\right) \operatorname{Vol}_{4}\left(R_{*}\right)
$$

where

$$
\operatorname{Vol}_{3}\left(R_{*}\right)=2 \pi^{2} b_{0}^{3}\left(g_{s} M\right)^{3 / 2} R_{*}^{3} \quad \operatorname{Vol}_{4}\left(R_{*}\right)=\frac{1}{2} \pi^{2} b_{0}^{4}\left(g_{s} M\right)^{2} R_{*}^{4} .
$$

denote, respectively, the 3 -volume of and the 4 -volume inside a 3 -sphere of radius $R_{*}$ as measured by the metric (36).

It now seems reasonable to assume that, when $p<<M$, the profile of the domain wall around $R=R_{*}$ approaches the supersymmetric configuration described in $\S 4.2$. Based on this intuition, we will set the domain wall tension $T_{\text {wall }}$ equal to its the supersymmetric value (57). Taking the leading order value $V_{\text {tot }}\left(\psi_{\text {min }}\right) \simeq 2 p \mu_{3} / g_{s}$ for the energy of the false vacuum, we thus get the following result for the classical action of the domain wall solution with radius $R_{*}$

$$
S\left(R_{*}\right)=\pi^{2} \mu_{3} b_{0}^{4} g_{s} M^{2}\left(M b_{0}^{2} R_{*}^{3}-p R_{*}^{4}\right)
$$

The two terms represent the two competing forces on the NS 5-brane domain wall bubble: the tension pulling it inward and the outward pressure caused by the lower energy of the supersymmetric vacuum inside the bubble. At the critical radius

$$
R_{*}=\frac{3 M b_{0}^{2}}{4 p}
$$

the two forces are balanced. Notice that, as expected, the domain wall becomes flat in the limit that $p / M$ approaches zero.

Plugging the critical value for $R_{*}$ back into the action, gives the final leading estimate of the nucleation rate

$$
\text { Decay rate } \simeq \exp \left(-\frac{27 b_{0}^{12} g_{s} M^{6}}{512 \pi p^{3}}\right)
$$

where we set $\mu_{3}=1 /(2 \pi)^{3}$. We see that the rate is very highly suppressed in the $p<<M$ regime we have been considering here.

In general, the above expression gives a negligible rate in the supergravity limit of large $M$ with $g_{s} M$ fixed. Note, however, that in our derivation of (65) we have assumed that $p / M$ is far below the critical value (about $8 \%$ in our probe approximation) where the false vacuum becomes classically unstable. We conclude that, by tuning $p / M$, we can make the non-supersymmetric vacuum arbitrarily long or short-lived. 


\section{$5 \quad$ Two Dual Perspectives}

In this section we consider two dual perspectives on our model. In particular, we propose a dual holographic description in terms of a nonsupersymmetric, metastable vacuum in the KS field theory. Secondly, as an additional motivation for this proposal, we summarize how our model may be described from the type IIA point of view.

\subsection{Holographic Dual Field Theory}

For simplicity, we first consider the case that $p=1$, which we expect to be related to the $S U(2 M-1) \times S U(M-1) \mathrm{KS}$ field theory. In addition to the $\mathcal{N}=1$ gauge multiplets, this theory has bifundamental fields, two in the $(2 M-1, \overline{M-1})$ representation and two in the $(\overline{2 M-1}, M-1)$ representation. So, from the perspective of the $S U(2 M-1)$, there are $N_{f}=2(M-1)$ flavors, one less than the number of colors. Hence it is no longer possible to write the color neutral baryonic combinations $\mathcal{B}$ and $\tilde{\mathcal{B}}$, and, as a result, the only supersymmetric vacuum of the system is the mesonic branch (16). This is the holographic dual of the stable vacuum with $M-1$ D3-branes, the situation we expect to land on after the decay process in the case $p=1$. The question now becomes, "Where do we find the other, nonsupersymmetric metastable vacuum corresponding to a single anti-D3 brane probing the conifold?"

Though qualitatively important, for large $M$, the presence of the single anti-D3 brane acts only as a small perturbation of the situation with $p=0$. (For example, the effective NS 5-brane potential $V_{\text {eff }}$ given in (45)-(40) has a perfectly smooth limit for $\frac{p}{M} \rightarrow 0$.) It seems reasonable to assume, then, that the nonsupersymmetric minimum for $p=1$ can be thought of as closely related to the baryonic vacuum of the supersymmetric theory with $p=0$. Indeed, as we have argued in $\S 4.2$, the NS 5 domain wall that separates it from the theory with $M-1 \mathrm{D} 3$-branes in the supersymmetric case represents a transition between the baryonic and mesonic branch.

We will now try to use this intuition to obtain a description of the nonsupersymmetric minimum. We introduce, in spite of the fact that $N_{f}=N_{c}-1$ in our case, the two "baryonic" superfields

$$
\mathcal{B}^{a}=\left(A_{1}\right)^{M-1}\left(A_{2}\right)^{M-1}, \quad \tilde{\mathcal{B}}^{a}=\left(B_{1}\right)^{M-1}\left(B_{2}\right)^{M-1},
$$

which are no longer colorless but carry a color index $a$ transforming in the fundamental representation of $S U(2 M-1)$. The idea is that the nonsupersymmetric theory corresponds to a false vacuum of the $p=1 \mathrm{KS}$ gauge theory characterized by a non-zero expectation value of these color charged "baryon" fields. Naturally, this will cost energy, but it seems a reasonable assumption that (for $p / M$ very small) this nonsupersymmetric vacuum may nonetheless be classically stable because it is separated from the supersymmetric minimum via a potential barrier. 
To make this proposal more concrete, let us derive the form of the superpotential of our model with $\mathcal{B}^{a}$ and $\tilde{\mathcal{B}}^{a}$ included. To this end, we start by adding to the $S U(2 M-1) \times S U(M-$ 1) gauge theory a single pair of scalar multiplets $A^{a}$ and $B^{a}$ (with $a$ denoting the $2 M-1$ color index), which we will then make very massive. The motivation for introducing these extra fields is that, before we decouple them, they augment the system to have $N_{f}=N_{c}$ so that we can introduce color neutral baryonic fields. Define the combination $\phi_{a b}=A_{a} B_{a}$. Now write the superpotential

$$
W=\lambda\left(N_{i j, \alpha \beta} N_{k l}^{\alpha \beta}\right) \epsilon^{i k} \epsilon^{j l}+X\left(\operatorname{det} N^{\prime}-\phi_{a b} \mathcal{B}^{a} \tilde{\mathcal{B}}^{b}-\Lambda^{4 M-2}\right)+m \operatorname{tr} \phi
$$

with $\operatorname{det} N^{\prime}$ the determinant of the $(2 M-1) \times(2 M-1)$ meson matrix obtained by including $A_{a}$ and $B_{a}$. Here the last term gives rise to a mass $m$ for the extra fields $A_{a}$ and $B_{a}$. We can decompose

$$
\operatorname{det} N^{\prime}=\left(\operatorname{tr} \phi+\phi_{a b} A_{i}^{\alpha, a} B_{j}^{\beta, b}\left(N^{-1}\right)_{\alpha \beta}^{i j}\right) \operatorname{det} N
$$

The lagrange multiplier term in (67) is the standard one for an $\mathcal{N}=1$ gauge theory with $N_{f}=N_{c}$. Notice that the extra field $\phi_{a b}$ does not appear in the first term in eqn (67); we omit it here to avoid a symmetry breaking expectation value for $\phi_{a b}$. Instead, we would like to keep $N_{c}$ equal to $2 M-1$ after integrating out $\phi_{a b}$. The supersymmetric $\phi_{a b}$ equations of motion now read

$$
m+X(\operatorname{det} N-\operatorname{tr} \mathcal{B} \tilde{\mathcal{B}})=0
$$

from which we can solve for $X$, and

$$
A_{i}^{\alpha, a} B_{j}^{\beta, b}\left(N^{-1}\right)_{\alpha \beta}^{i j} \operatorname{det} N=\mathcal{B}^{a} \tilde{\mathcal{B}}^{b}
$$

Inserting the solution for $X$ back into $W$ gives the superpotential (with $p=1$ )

$$
W=\lambda\left(N_{i j}\right)_{\beta}^{\alpha}\left(N_{k \ell}\right)_{\alpha}^{\beta} \epsilon^{i k} \epsilon^{j \ell}+p\left(\frac{\Lambda_{1}^{4 M-p}}{\operatorname{det}_{i j, \alpha \beta} N-\operatorname{tr} \mathcal{B} \tilde{\mathcal{B}}}\right)^{\frac{1}{p}}
$$

with $\Lambda_{1}^{4 M-1}=m \Lambda^{4 M-2}$. This is our proposed superpotential of the theory with the "baryonic" superfields present.

In case of general $p$, we can similarly write color charged "baryon" fields, which transform in the $p$-th anti-symmetric product of the fundamental. Although we have not done the explicit analysis in this general case, a natural guess is that the superpotential will take the form (71). As a primitive reasonability check, we note that the supersymmetric equation of motion for the "baryon" field, $\partial_{\mathcal{B}} W=0$, yields the condition that $\mathcal{B}^{a}=\tilde{\mathcal{B}}^{a}=0$, so the mesonic vacuum remains present as the only supersymmetric vacuum, according with our expectations. 
In general, without more detailed control over the dynamics, the superpotential on its own provides at most inconclusive evidence of the possible existence of other, nonsupersymmetric vacua of the theory. Still, if our proposal is right, it should at least give some hint. A general comment: Formally, the equation $\partial_{\mathcal{B}} W=0$ also admits one other solution, namely $\operatorname{tr} \mathcal{B} \tilde{\mathcal{B}} \rightarrow \infty$. While it is of course dangerous to suggest that this implies the existence of another supersymmetric vacuum, it does indicate that, as a function of the baryon condensate, the full potential $V=|d W|^{2}$ of the theory will have a maximum at some intermediate scale (which one would expect to be near $\operatorname{tr} \mathcal{B} \tilde{\mathcal{B}} \sim \Lambda_{1}^{4 M-4 p}$ ). It is conceivable, therefore, that there exists another minimum at large $\operatorname{tr} \mathcal{B} \tilde{\mathcal{B}}$.

The strongest evidence for the existence of the nonsupersymmetric "baryonic" vacuum, however, still comes from the supergravity analysis. The characterization of the nonsupersymmetric model in terms of $p$ anti-D3 branes inside the conifold geometry suggests that, somewhere in the dual field theory, there should be a hint of an (unbroken) $S U(p)$ gauge symmetry. Indeed, our proposed dual interpretation in terms of a phase with a non-zero condensate for $\operatorname{tr} \mathcal{B} \tilde{\mathcal{B}}$ naturally leads to a breaking of the $S U(2 M-p)$ gauge symmetry to $S U(p)$. It seems natural to identify the worldvolume theory of the $p$ anti-D3 branes with the effective low energy description of this $S U(p)$ sector. In the following subsection, we will find an independent indication from the type IIA perspective that the nonsupersymmetric theory is described by an $S U(p) \times S U(M-p)$ gauge theory.

\subsection{Type IIA Brane Configurations}

The RG cascade in the KS system can also be understood via a dual type IIA perspective [2]. In the IIA description, one studies a theory on D4 branes suspended between NS 5 branes. Consider an NS 5 and an NS 5' brane, the first filling out the 012345 directions, and the latter the 012389 directions in spacetime. Suppose them separated only along the $x^{6}$ direction, compactified on a circle. One can then stretch $N$ D4 branes around the circle, and $M \mathrm{D} 4$ branes on one of the two segments. The former correspond to the $N$ D3 branes and the latter to fractional branes. The resulting field theory has $S U(N+M) \times S U(N)$ gauge group and the matter stretching across the NS 5 and NS $5^{\prime}$ branes gives precisely the bi-fundamentals which arise in the KS field theory.

In this description, the forces on the branes are not perfectly balanced - the NS branes bend together on the segment with the additional fractional branes. This corresponds to the fact that the $S U(N+M)$ gauge theory has $N_{f}=2 N$ flavors and becomes strongly coupled as one flows to the IR. One can move the $5^{\prime}$ brane through the NS 5 brane and around the $x^{6}$ circle to avoid this intersection; this will have the effect of reducing the gauge group to $S U(N) \times S U(N-M)$. This is the first step in the RG cascade, and it is repeated until the rank of the gauge groups is low enough that $N_{f}<N_{c}$ in one of the factors and the non-perturbative dynamics becomes more subtle.

The difference in our setup is the addition of $p \overline{D 3}$ branes to the conifold. In the dual brane configuration, these should appear as $p \overline{D 4}$ branes stretched between the NS 5 and 
the NS $5^{\prime}$ branes in addition to those already present in the KS setup. There are now (at least) two obvious options:

- The $p$ anti-branes can annihilate with the D4 branes in each segment, leaving an $S U(N+$ $M-p) \times S U(N-p)$ gauge theory. This subsequently undergoes the RG cascade as described above; assuming $N=K M$ and $p<<M$, the endpoint is a supersymmetric $S U(2 M-p) \times$ $S U(M-p)$ gauge theory. This is, of course, the dual gauge theory description of the final state $(7)$.

- Alternatively, one can first go through the KS RG cascade with the D4 branes, leaving a pure $S U(M)$ gauge theory from the D4 brane sector. Then, including the $p$ anti-branes, one finds a nonsupersymmetric theory with gauge group $S U(M-p) \times S U(p)$. Special cases of this theory were discussed in [18]. This is a type IIA dual description of our nonsupersymmetric configuration. It would be interesting to understand the vacuum structure and gauge symmetry breaking patterns found in $\S 3$ from the IIA perspective. The analysis in [18] finds evidence of a symmetry breaking pattern which depends sensitively on the radius of the $x_{6}$ circle, but is carried out far from the $p<<M, N$ regime of interest to us here.

\section{Concluding Remarks}

We have found that the configuration of $p \overline{D 3}$ branes probing the KS geometry constitutes a rich system with several interesting properties. The basic physics is apparent in Figure 4. For $p<<M$, the system relaxes to a metastable nonsupersymmetric vacuum but eventually tunnels to a supersymmetric final state. This decay has a strongly suppressed rate given in (65) and the nonsupersymmetric vacuum can therefore be made arbitrarily long-lived. For $p / M$ larger than some critical value (of about $8 \%$ in our probe approximation) the decay takes place classically. In both cases, the decay is effected by "brane/flux annihilation" where the branes first form an NS 5-brane which later unwinds around the $S^{3}$, creating $M-p$ D3 branes in the process. An important remaining problem is to find a supergravity solution of the nonsupersymmetric minimum that includes the backreaction of the $p$ NS 5 -branes.

The same basic brane/flux transmutation process whereby fluxes are traded for D3 branes may also provide a new perspective on many of the dualities currently under study as geometric transitions. An analogue of our microscopic description of this process via NS 5 brane nucleation may also play an important role in those transitions, which encode the information about D-brane gauge theories in terms of dual geometries with fluxes (see e.g. $[4,20])$.

In most of this paper, we have restricted our attention to the infrared physics of the model, implicitly assuming that it embedded in a non-compact warped geometry with fixed boundary conditions in the UV. It is an interesting question, however, to ask what happens 

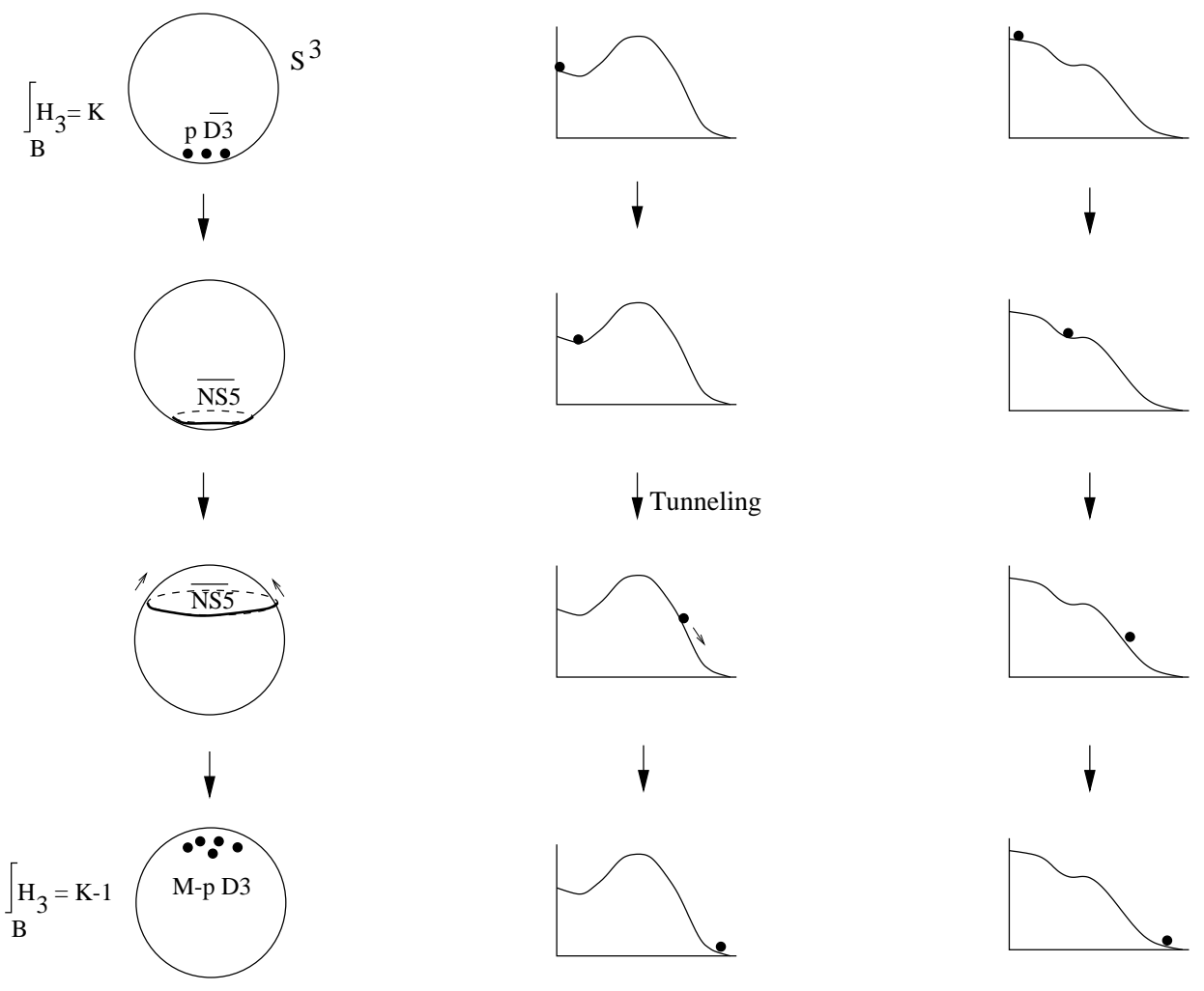

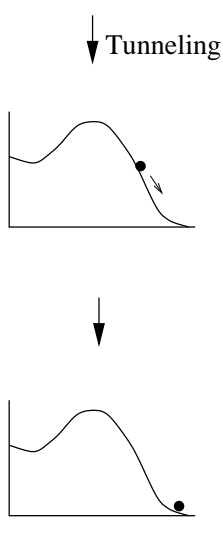

(i)

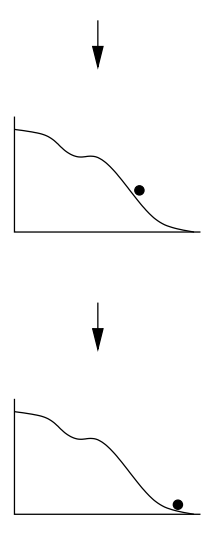

( i i )

Fig 4:Schematic depiction of the brane/flux annihilation process for (i) subcritical and (ii) supercritical values for $p / M$.

when we embed our model in a true string compactification with a finite volume as constructed in [5]. In this case, the holographically dual gauge theory will be coupled to 4-d gravity. Looking at the form of the potentials in figure 4 , it is then natural to ask what type of cosmological evolutions are possible in this set-up.

Besides the $\psi$ field, the string compactification will generally give rise to many other light moduli fields. In the basic model of [5], all can be made massive except for the Kähler modulus $u(x)$ controlling the overall volume of the $6 \mathrm{~d}$ internal space $Y$. If we ignore the backreaction due to the branes and fluxes, it is defined by

$$
d s^{2}=g_{\mu \nu} d x^{\mu} d x^{\nu}+e^{2 u} g_{i \bar{j}} d z^{i} d \bar{z}^{\bar{j}}
$$

where $d s^{2}$ is the $10 \mathrm{~d}$ string frame metric and $g_{i \bar{j}}$ the Ricci-flat metric on $Y$. Following the steps outlined in [5], one obtains for the $4 \mathrm{~d}$ low energy effective action

$$
S=\frac{1}{2 \kappa_{4}^{2}} \int d^{4} x\left(-\tilde{g}_{4}\right)^{1 / 2}\left(\tilde{R}_{4}-6\left(\partial_{\mu} u\right)^{2}-a_{0}^{2} e^{-6 u} V_{2}(\psi)\left(\partial_{\mu} \psi\right)^{2}+a_{0}^{4} e^{-12 u} V_{\text {tot }}(\psi)\right)
$$


with $\tilde{g}_{4}$ the $4 \mathrm{~d}$ Einstein frame metric, and $V_{2}$ and $V_{\text {tot }}$ as in (40) and (45)-(49). The coefficient $a_{0}$ is the warpfactor at the location of the anti-D3-branes.

The dynamics of this low energy field theory is dominated by the steep inverse-volume dependence evident in (73), which implies that the presence of the extra energy density in the anti-branes will quickly force the Calabi-Yau manifold to decompactify. In addition, it prevents the model from giving rise to any appreciable inflation. To stop this decompactification process, or to get an inflationary solution, it seems that one would have to find a novel means of stabilizing the Kähler moduli (for a discussion of moduli stabilization in roughly this context, see e.g. [5, 19]).

The gravitational effect of brane nucleation processes that induce discrete flux jumps has recently been investigated in [21], as a possible dynamical mechanism for neutralizing the cosmological constant. In particular the set-up considered in the second reference appears closely related to ours.

\section{Acknowledgements}

We would like to thank A. Bergman, C. S. Chan, K. Dasgupta, S. Giddings, C. Herzog, I. Klebanov, J. March-Russell, J. Maldacena, S. Mukhi, P. Ouyang, J. Polchinski, M. Schulz, N. Seiberg, M. Strassler, S. Trivedi, E. Verlinde and E. Witten for helpful discussions. The work of J.P. and H.V. is supported in part by a National Science Foundation grant 98-02484 and an NSF Graduate Research Fellowship. The work of S.K. is supported in part by a Packard Fellowship, a Sloan Fellowship, the DOE under contract DE-AC03-76SF00515, and National Science Foundation grant PHY-0097915.

\section{References}

[1] For a review, see: O. Aharony, S. Gubser, J. Maldacena, H. Ooguri and Y. Oz, "Large N Field Theories, String Theory and Gravity," Phys. Rept. 323, 183 (2000) [hepth/9905111].

[2] I. Klebanov and M. Strassler, "Supergravity and a Confining Gauge Theory: Duality Cascades and $\chi$ SB Resolution of Naked Singularities," JHEP 0008, 052 (2000) [hepth/0007191].

[3] J. Maldacena and C. Nunez, "Towards the Large N Limit of Pure $\mathcal{N}=1$ SuperYangMills," Phys. Rev. Lett. 86, 588 (2001) [hep-th/0008001].

[4] C. Vafa, "Superstrings and Topological Strings at Large N," J. Math. Phys. 42, 2798 (2001) [hep-th/0008142]. 
[5] S. Giddings, S. Kachru and J. Polchinski, "Hierarchies from Fluxes in String Compactifications," [hep-th/0105097].

[6] L. Randall and R. Sundrum, "A Large Mass Hierarchy from a Small Extra Dimension," Phys. Rev. Lett. 83, 3370 (1999) [hep-th/9905221].

[7] H. Verlinde, "Holography and Compactification," Nucl. Phys. B580, 264 (2000) [hepth/9906182];

C. Chan, P. Paul and H. Verlinde, "A Note on Warped String Compactification," Nucl. Phys. B581, 156 (2000) [hep-th/0003236].

[8] P. Mayr, "On Supersymmetry Breaking in String Theory and its Realization in Brane Worlds," Nucl. Phys. B593, 99 (2001) [hep-th/0003198];

P. Mayr, "Stringy World Branes and Exponential Hierarchies," JHEP 0011, 013 (2000) [hep-th/0006204].

[9] K. Becker and M. Becker, "M-theory on Eight Manifolds," Nucl. Phys. B477, 155 (1996) [hep-th/9605053];

K. Becker and M. Becker, "Supersymmetry Breaking, M-theory and Fluxes," JHEP 0107, 038 (2001) [hep-th/0107044].

[10] S. Gukov, C. Vafa and E. Witten, "CFTs from Calabi-Yau Fourfolds," Nucl. Phys. B584, 69 (2000) [hep-th/9906070];

K. Dasgupta, G. Rajesh, and S. Sethi, "M-theory, Orientifolds and G-flux," JHEP 9908, 023 (1999) [hep-th/9908088];

B. Greene, K. Schalm and G. Shiu, "Warped Compactifications in M and F-theory," Nucl. Phys. B584, 480 (2000) [hep-th/0004103].

[11] G. Curio, A. Klemm, D. Lust and S. Theisen, "On the Vacuum Structure of Type II String Compactifications on Calabi-Yau Spaces with H Fluxes," Nucl. Phys. B609, 3 (2001) [hep-th/0012213];

G. Curio, A. Klemm, B. Kors and D. Lust, "Fluxes in Heterotic and Type II String Compactifications," [hep-th/0106155].

[12] C. Herzog, I. Klebanov and P. Ouyang, "Remarks on the Warped Deformed Conifold," [hep-th/0108101].

[13] N. Seiberg, "Exact Results on the Space of Vacua of Four-Dimensional SUSY Gauge Theories," Phys. Rev. D49, 6857 (1994) [hep-th/9402044].

[14] J. Polchinski and M. Strassler, "The String Dual of a Confining Four-Dimensional Gauge Theory," [hep-th/0003136].

[15] R. Myers, "Dielectric Branes," JHEP 9912, 022 (1999) [hep-th/9910053]. 
[16] J. Maldacena and H. Nastase, "The Supergravity Dual of a Theory with Dynamical Supersymmetry Breaking," JHEP 0109, 024 (2001) [hep-th/0105049].

[17] S. Coleman, "The Fate of the False Vacuum I. Semiclassical Theory," Phys. Rev. D15, 2929 (1977).

[18] S. Mukhi and N. Suryanarayana, "A Stable Non-BPS Configuration from Intersectiong Branes and Anti-branes," JHEP 0006, 001 (2000) [hep-th/0003219].

[19] E. Silverstein, “(A)dS Backgrounds from Asymmetric Orientifolds," [hep-th/0106209].

[20] F. Cachazo, K. Intriligator and C. Vafa, "A Large N Duality via a Geometric Transition," Nucl. Phys. B603, 3 (2001) [hep-th/0103067];

F. Cachazo, B. Fiol, K. Intriligator, S. Katz and C. Vafa, "A Geometric Unification of Dualities," [hep-th/0110028].

[21] R. Bousso and J. Polchinski, "Quantization of Four-form Fluxes and Dynamical Neutralization of the Cosmological Constant" JHEP 0006006 (2000) [hep-th/0004134];

J.L. Feng, J. March-Russell, S. Sethi and F. Wilczek, "Saltatory Relaxation of the Cosmological Constant," Nucl.Phys. B602 307 (2001) [hep-th/0005276] 